\title{
Closed-form analytical expressions for the potential fields generated by triangular monolayers with linearly distributed source strength
}

\author{
Adriaan van Oosterom
}

Received: 2 August 2011 / Accepted: 10 October 2011/Published online: 28 October 2011

(C) The Author(s) 2011. This article is published with open access at Springerlink.com

\begin{abstract}
The solution of the mixed boundary value problem of potential theory involves the computation of the potential field generated by monolayer and double layer source distributions on surfaces at which boundary conditions are known. Closed-form analytical expressions have been described in the literature for the potential field generated by double layers having a linearly distributed strength over triangular source elements. This contribution presents the corresponding expression for the linearly distributed monolayer strength. The solution is shown to be valid for all observation points in space, including those on the interior, edges and vertices of the source triangle.
\end{abstract}

Keywords Boundary element method - Bioelectricity · Linear shape functions

\section{Introduction}

The boundary element method (BEM) is a well-known method for computing the (quasi-static) potential field resulting from applied forces on the boundaries of piecewise homogeneous media, as well as within them. Examples of applications are those in electrostatics, deformations in elastic media, and in bioelectricity. The basis of the method was formulated by Smythe [27]. Theory and

This article is dedicated to the memory of Prof. dr. Jan Strackee, whose interest in the topic addressed was the inspiration for its completion.

A. van Oosterom $(\bowtie)$

Radboud University Nijmegen, Nijmegen, The Netherlands

e-mail: avo-linden@home.nl examples of 2D applications can be found, in, e.g., [4]. Early applications to 3D are described in, e.g. [3, 10, 16], more recent ones in $[12,23,32]$.

In bioelectricity, the applied forces result from impressed electric currents and the medium is a volume conductor model comprising a set of closed non-intersecting surfaces that are nested inside the body surface. The surfaces considered are the interfaces between regions having a different electric conductivity. The surfaces carry so-called secondary sources. These are virtual sources that are placed in a virtual, homogeneous, isotropic medium of infinite extent. Their strengths are computed such that the continuity conditions of electric volume conduction theory at these interfaces are satisfied [12, 22, 27].

The requisite basic computations involved in the BEM are those of the potential fields generated by monolayer and/or double layer sources distributed over the surfaces. Based on the superposition theorem, the potential at each field point is computed as the sum of the contributions of the primary sources and those of the secondary current sources on all of the small surface elements considered.

Prominent applications of the BEM in bioelectricity aim at linking the potential field on a closed internal surface that encompasses all primary sources to the potential field on the thorax surface. Examples are the ones in which the internal surface is the pericardium, the surface closely encompassing the heart [3, 5, 13, 17, 25, 29], or the cortical surface [2]. The solving of this type of mixed boundary value problem (Cauchy problem [27]) involves both double and monolayers [3, 20, 27, 29].

The interfaces that are relevant in the field of bioelectricity generally have a complex shape. This necessitates a numerical handling of the computation of the fields generated by the sources, both the primary and the secondary ones [13]. To this end, the surfaces are subdivided into 
numerous, small planar elements. The triangular shape produces a close fit of the mesh to the interfaces.

In early applications, the distribution of the source strength over the triangle, the so-called shape function, was taken to be uniform. However, this approach was soon realized as being suboptimal since it creates an unrealistic discontinuity of the source strength across the edges of neighboring triangles. Instead, linear shape functions with their strength at their vertices proportional to the local potential have been proposed. Closed-form analytical expressions are known for the potential fields generated by source distributions on a triangle of the uniform double layer [30], the linearly distributed variant [6] as well as for the uniform monolayer [9].

Up until now, the field generated by the monolayer with linearly distributed source strength has been computed on the basis of numerical methods for solving the involved integral. This article presents an exact, closed form expression for this solution.

The organization of the article is as follows. First, in Sect. 2, the basic notations used are listed, followed by the introduction to the problem in hand. Next, a section is included that describes the solution for the uniform monolayer [9] in a notation that prepares the way for the derivation for the linearly distributed monolayer presented in the subsequent section.

Next, some examples are shown of the resulting potential fields, including those for the situation in which a field point coincides with one of the vertices of the source triangle. This is the situation that is highly relevant in BEM-based computations.

A further motivation for this contribution and the relevance of the results is discussed in the final section. In an appendix, the basic integrals used are listed. Moreover, for the sake of completeness, the corresponding results for the double layer are included, taken from the literature, but now cast in the notation of this article.

\section{Preliminaries}

\subsection{Notation}

Throughout, vectors in 3D space are denoted by lower case variables with an overhead arrow, e.g., $\vec{r}$, their lengths (unit $\mathrm{m})$ by dropping the arrow. The vector product of vectors $\vec{a}$ and $\vec{b}$ is denoted by $\vec{a} \times \vec{b}$ (cross product), the scalar vector product by $\vec{a} \cdot \vec{b}$ (dot product). The source triangle is denoted by $\Delta$; its normal $\vec{n}$ is found from the cross product of any two of its edges. The norm of $\vec{n}$, denoted by $n$, equals twice the area $\left(S_{\Delta}\right)$ of the source triangle. The normalized version of $\vec{n}$ is denoted by $\vec{n}_{\mathrm{n}}$.
Variables expressed in the domain of linear algebra are denoted as follows. Column vectors are shown in lowercase bold type face, e.g., applied to $\vec{n}$ this yields $\mathbf{n}$. Row vectors are primed. Vector norms are shown using regular font. Column vectors having unit elements only are denoted by $\mathbf{u}$.

Matrices are shown in upper-case bold. The transpose of a matrix $\mathbf{M}$ is primed: $\mathbf{M}^{\prime}$.

\subsection{Problem statement}

The fields produced by the sources on the basic, triangular BEM element $\Delta$ to be discussed are the ones generated by current source densities impressed over $\Delta$. The pertinent geometrical configuration is depicted in Fig. 1. The triangle vertices are labeled $(k, l, m)$ in a clockwise order when viewed from the origin. The normal of the triangle is oriented in the direction of a right-hand screw rotated in the order of the vertices $k, l, m$. The edges of the triangle are defined as $\vec{e}_{k}=\vec{r}_{\ell}-\vec{r}_{k}, \vec{e}_{\ell}=\vec{r}_{m}-\vec{r}_{\ell}$, and $\vec{e}_{m}=\vec{r}_{k}-\vec{r}_{m}$.

\section{The potential field generated by impressed monolayer current distributions}

The problem addressed in this article is the computation of the potential $\Phi\left(\vec{r}^{\prime}\right)$ at field point $\vec{r}^{\prime}$ resulting from a monolayer with strength $J_{\Delta}(\vec{r})$ (unit $\mathrm{A} / \mathrm{m}$ ) that impresses a point source type of electric current $I(\vec{r})=J_{\Delta}(\vec{r}) \mathrm{d} S$ (unit A) into the surrounding medium from an infinitesimally small part of the triangle, $\mathrm{d} S$, located at $\vec{r}$. In an analogy with Coulomb's law for point charges in infinite space [27],

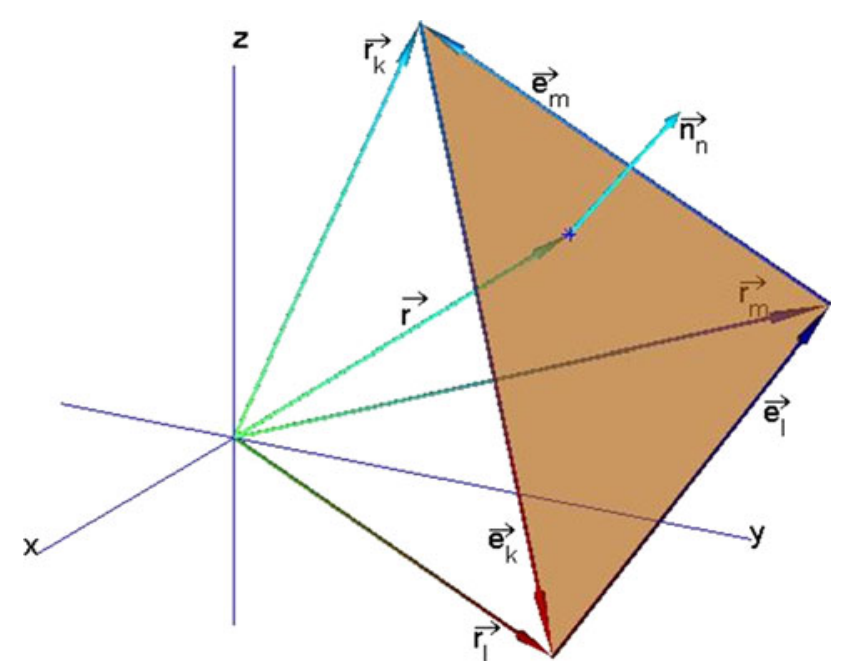

Fig. 1 Diagram introducing the computation of the potential at the origin generated by current source densities over a triangle. The vector $\vec{r}$ points from the field point, here taken to be the origin, to source locations on the triangle 
the potential field in the electric volume conduction problem is found from the superposition of the contributions of the elementary sources:

$\Phi\left(\vec{r}^{\prime}\right)=\frac{1}{4 \pi \sigma} \int_{\Delta} \frac{J_{\Delta}(\vec{r})}{R} \mathrm{~d} S$,

with $\sigma$ the electric conductivity of the medium (unit $\mathrm{S} / \mathrm{m}$ ) and $R$ the length of the vector connecting field point $\vec{r}^{\prime}$ to source location $\vec{r}$.

The solution for an arbitrary field point is identical to the one found by shifting the entire geometry such that the field point of interest lies at the origin. By doing so, Eq. 1 may be written as

$\Phi=\frac{1}{4 \pi \sigma} \int_{\Delta} \frac{J_{\Delta}(\vec{r})}{r} \mathrm{~d} S$,

with $\Phi=\Phi\left(\vec{r}^{\prime}=\overrightarrow{0}\right)$.

\subsection{The uniform monolayer}

For a uniform monolayer we have $J_{\Delta}(\vec{r})=J_{\Delta}$ and Eq. 2 reduces to

$\Phi=\frac{1}{4 \pi \sigma} \int_{\Delta} \frac{J(\vec{r})}{r} \mathrm{~d} S=\frac{J_{\Delta}}{4 \pi \sigma} \int_{\Delta} \frac{1}{r} \mathrm{~d} S$.

The basic problem to be addressed is the solving of the integral on the right, the factor scaling it being application specific. In the literature, a multitude of papers dealing with this the solution of the integral are documented $[9,11,14$, $15,18,19,24]$, exhibiting different approaches to the solution method and details of the results. Below, the solution derived by Ferguson et al. [9] is presented in a compact notation that is used throughout this article. The solution, denoted as $\Gamma_{\Delta}$, reads

$\Gamma_{\Delta}=h \Omega_{\Delta}+\mathrm{h}^{\prime} \gamma$.

The variables shown represent:

- $h$ : the height of the tetrahedron formed by the three vectors defining the triangle and the field point (at the origin) as shown in Fig. 1, computed from $h=\left[\vec{r}_{k} \vec{r}_{l} \vec{r}_{m}\right] / n$, with $\left[\vec{r}_{k} \vec{r}_{l} \vec{r}_{m}\right]=\vec{r}_{k} \times \vec{r}_{\ell} \cdot \vec{r}_{m}$ denoting the triple vector product of the vectors specifying the triangle vertices relative to the field point. In the sequel this triple vector product is denoted by $T$. Its numerical value equals that of the determinant of the matrix of size $3 \times 3$ whose elements are the vertex coordinates of the source triangle.

- $\Omega_{\Delta}$ : the solid angle subtended by the source triangle at the field point (Eq. 25).

- h: a column vector with elements: $h_{k}=\left[\vec{r}_{k} \vec{r}_{l} \vec{n}_{\mathrm{n}}\right] / e_{k}$, $h_{\ell}=\left[\vec{r}_{\ell} \vec{r}_{m} \vec{n}_{\mathrm{n}}\right] / e_{\ell}$, and $h_{m}=\left[\vec{r}_{m} \vec{r}_{k} \vec{n}_{\mathrm{n}}\right] / e_{m}$. These represent the signed distances between the projection, $\vec{p}$, of the field point on the plane of the source triangle and its projections on the lines carrying the edges. The sign of the elements is negative if, when traveling along the line segment in the direction of the corresponding edge, the projection $\vec{p}$ lies on the right, else it is positive except if $\vec{p}$ lies on the line segment, where it is zero.

- $\gamma$ : a column vector with elements $\gamma_{j}(j=k, l, m)$ that are the line integrals over the edges $j$ (Eq. 19).

An alternative derivation of Eq. 4, in which the solid angle appearing in this expression is explained, is shown in [28].

\section{The potential field of linearly distributed monolayer current densities}

We now turn to the main topic, the handling of the case where the monolayer strength is linearly distributed over the triangle. To this end, the monolayer strength is written as

$$
\begin{aligned}
J_{\Delta}(\vec{r})= & J\left(\vec{r}_{k}\right) w_{k}(\vec{r})+J\left(\vec{r}_{\ell}\right) w_{\ell}(\vec{r}) \\
& +J\left(\vec{r}_{m}\right) w_{m}(\vec{r}),
\end{aligned}
$$

with (dimensionless) linear shape functions $w_{j}(\vec{r}), j \in$ $(k, \ell, m)$ having a unit value at any vertex $j$ and zero value at the remaining two.

Inserting Eq. 5 in 2 yields

$\Phi=\frac{1}{4 \pi \sigma} \sum_{j=k, \ell, m} J\left(\vec{r}_{j}\right) \int_{\Delta} \frac{w_{j}(\vec{r})}{r} \mathrm{~d} S$,

showing the integral in Eq 2 as broken up into three subintegrals of identical type.

A function having the desired nature of $w_{j}(\vec{r})$ for $j=k$ is

$w_{k}(\vec{r})=\frac{\left[\vec{r}_{\ell} \vec{r}_{m} \vec{r}\right]}{\left[\vec{r}_{\ell} \vec{r}_{m} \vec{r}_{k}\right]}=\frac{\vec{r}_{\ell} \times \vec{r}_{m} \cdot \vec{r}}{T}$.

The other two are found by cyclic permutation of the indices $k, \ell$, and $m$. Using $\vec{r}_{k} \times \vec{r}_{\ell}+\vec{r}_{\ell} \times \vec{r}_{m}+\vec{r}_{m} \times \vec{r}_{k}=\vec{n}$, a property of the tetrahedron, it can be seen that

$\sum_{j=k, \ell, m} w_{j}(\vec{r})=1$,

as required.

In the following, the integral in Eq. 6 for index $j \in$ $(k, \ell, m)$ is denoted by $\Gamma_{j}$, a scalar (unit $\mathrm{m}$ ), and its vector notation by a column vector $\Gamma$. By introducing $\vec{z}_{k}=\vec{r}_{\ell} \times \vec{r}_{m}$, we may write $\Gamma_{j}$ as

$\Gamma_{j}=\frac{1}{T} \vec{z}_{j} \cdot \int_{\Delta} \underset{r}{r} \mathrm{~d} S$.

Based on Eq. 8 and the additive property of integration, the terms $\Gamma_{j}$ must add up to the integral for the uniform distribution, $\Gamma_{\Delta}$ : 
$\Gamma_{k}+\Gamma_{\ell}+\Gamma_{m}=\Gamma_{\Delta}$.

Next, an auxiliary vector $\vec{G}$ (unit $\mathrm{m}^{2}$ ) is introduced:

$\vec{G}=\int_{\Delta} \frac{\vec{r}}{r} \times \mathrm{d} \vec{S}=-\int_{\Delta} \nabla r \times \mathrm{d} \vec{S}=-\oint r \mathrm{~d} \vec{c}$,

in which the equality on the right follows from Stoke's theorem [27]. The value of the contour integral is

$$
\begin{aligned}
\vec{G} & =\oint r \mathrm{~d} \vec{c}=\sum_{j=k, \ell, m} \int_{0}^{1} r(\lambda) \vec{e}_{j} \mathrm{~d} \lambda \\
& =\sum_{j=k, \ell, m} \frac{\vec{e}_{j}}{e_{j}} \int_{0}^{1} r(\lambda) e_{j} \mathrm{~d} \lambda=\sum_{j=k, \ell, m} \frac{\vec{e}_{j}}{e_{j}} I_{1, j}
\end{aligned}
$$

with $I_{1, j}$ denoting the line integral $I_{1}$ (Eq. 21) pertaining to edge $j$; its column vector variant is denoted as $\mathbf{I}_{1}$.

By inserting the vector synthesis $\vec{r}=\frac{1}{T} \sum_{j=k, \ell, m}\left(\vec{z}_{j} \cdot \vec{r}\right) \vec{r}_{j}$ in the left integral in Eq. 11, an alternative for $\vec{G}$ is found:

$$
\begin{aligned}
& \vec{G}=\frac{1}{T} \int_{\Delta} \sum_{j=k, \ell, m} \frac{\left(\vec{z}_{j} \cdot \vec{r}\right) \vec{r}_{j}}{r} \times \vec{n}_{n} \mathrm{dS} \\
& =\vec{n}_{n} \times \sum_{j=k, \ell, m} \vec{r}_{j} \frac{1}{T} \int_{\Delta} \frac{\left(\vec{z}_{j} \cdot \vec{r}\right)}{r} \mathrm{dS} .
\end{aligned}
$$

By recognizing $\Gamma_{j}$ as introduced in Eq. 9, we have

$$
\vec{G}=\vec{n}_{\mathrm{n}} \times \sum_{j=k, \ell, m} \vec{r}_{j} \Gamma_{j}
$$

The vectors $\vec{n}_{\mathrm{n}} \times \vec{r}_{j}$ are stored as the columns of a matrix $\mathbf{N}$ of size $3 \times 3$ and a column vector $\mathbf{y}$ is defined by its elements $\sum_{j=k, \ell, m} \frac{\vec{e}_{j}}{e_{j}} I_{1, j}$. Equating the final expression for $\vec{G}$ shown in Eq. 12 to the one in Eq. 13 yields $\mathbf{N} \boldsymbol{\Gamma}=\mathbf{y}$, from which it may seem as if the solution $\Gamma$ might be found. However, the rank of matrix $\mathbf{N}$ is 2 since the vectors $\vec{n}_{\mathrm{n}} \times$ $\vec{r}_{j}$ lie in the same plane, a plane normal to $\vec{n}_{\mathrm{n}}$. Hence, the linear system is under-determined. This problem is dealt with by adding Eq. 10 as a constraint, which leads to the $4 \times 3$ linear system

$$
\left[\begin{array}{c}
\mathbf{N} \\
\mathbf{u}^{\prime}
\end{array}\right] \boldsymbol{\Gamma}=\left[\begin{array}{r}
\mathbf{y} \\
\Gamma_{\Delta}
\end{array}\right],
$$

The least squares solution of this system yields the numerical column vector $\boldsymbol{\Gamma}$,

$\boldsymbol{\Gamma}=\left(\mathbf{Z}^{\prime} \mathbf{n} \Gamma_{\Delta}-\mathbf{E}_{\mathrm{c}}^{\prime} \mathbf{E}_{\mathrm{n}} \mathbf{I}_{1}\right) / n$,

in which $\mathbf{E}_{\mathrm{c}}$ is a matrix whose columns are coordinates of the edges of the source matrix after one step of cyclic permutation, i.e., $\left[\mathbf{e}_{\ell} \mathbf{e}_{m} \mathbf{e}_{k}\right]$, and $\mathbf{E}_{\mathrm{n}}$ a matrix whose columns are the normalized edge vectors.
The three elements of $\boldsymbol{\Gamma}$ are the three integrals in Eq. 6, thus yielding the final solution:

$\Phi=\frac{1}{4 \pi \sigma} \sum_{j=k, \ell, m} J\left(\vec{r}_{j}\right) \Gamma_{j}$

resulting from combining Eqs. 6 and 16, an expression that holds true throughout 3D space.

\section{Results}

The most pertinent result discussed in this article is the closed form analytical expression Eq. 15, which, inserted in Eq. 16, specifies the potential field generated by a current monolayer over a triangle with linearly distributed source density. Throughout, the pertinent variables are expressed in SI units.

\subsection{Numerical aspects}

When testing numerical implementation of Eq. 16 the computation of the $\gamma_{j}$ factors, introduced in Eq. 4 and specified in Eq. 19, proved to be the most sensitive element. As discussed in Appendix 1, and based on the physics involved, the argument of the logarithm must be greater than one. To ensure this property in the wake of rounding off steps in the numerical handling, the machine epsilon was added to the numerator as well as to the denominator in Eq. 19. Following this, for field points in the plane of the source triangle, on its edges, at its vertices as well as for field points very close to it, the results proved to be accurate within machine precision.

\subsection{Some examples}

In the following, some examples are presented of potential fields generated based on Eq. 16, also aimed at demonstrating the major properties of these fields. In these examples, a unit value of the conductivity $\sigma$ is assigned to the medium.

Equation 16 was stated to be valid throughout 3D space. This is illustrated in Fig. 2: the potential field in the plane of the triangle. The $\left[\begin{array}{ll}x y & y\end{array}\right]$ vertex coordinates are: A: [2 000$], \mathrm{B}$ : $[-1.520]$ and $C:[-100]$. The left panel depicts the situation in which the linear distribution of the source current density was specified by assigning a unit value $\left(J=1 \mathrm{~A} / \mathrm{m}^{2}\right)$ to vertex A and zero values to the other two. The resulting field generated by the uniform unit source density is shown on the right panel. The nature of the fields resulting from assigning a unit density to either vertex $\mathrm{B}$ or $\mathrm{C}$ is similar to the ones shown in the left panel. The potential values at the vertices 
Fig. 2 Left panel potential field in plane of a monolayer linearly distributed over a triangle, with unit source density $J$ at vertex A and zero at vertices $\mathrm{B}$ and $\mathrm{C}$; iso-potential line spacing 0.025 , maximum: 0.18814. Right panel corresponding result for uniform source strength. Isopotential line spacing 0.05 , maximum: 0.45853 . Location of the maxima marked by + . Values at the vertices as listed in Table 1

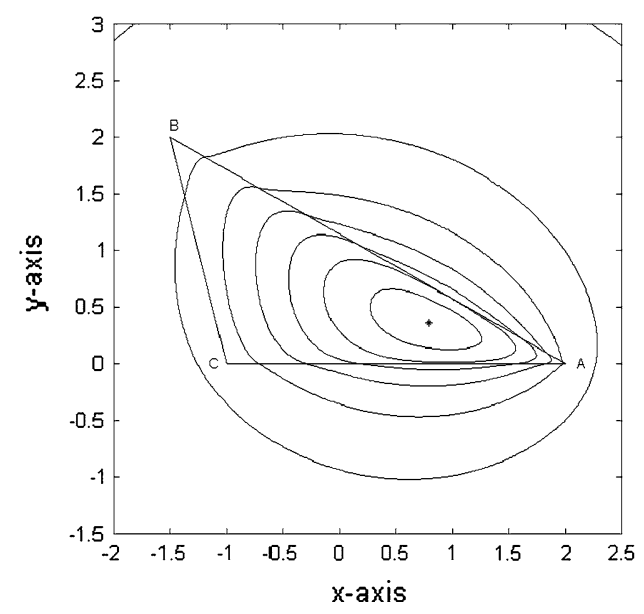

for the three basic linearly distributed cases and for the uniform case, as well as the corresponding extremes are listed in Table 1.

The corresponding fields on the plane $z=0.5$ are shown in Fig. 3.

Another view on the nature of the potential field of the monolayer is presented in Fig. 4. It depicts the potential profile along a line directed along the normal of the source triangle (parallel to the $z$-axis), passing through its center of gravity. The profile is generated by a uniform density $J_{\Delta}$ over the triangle (right panel Fig. 2). A characteristic feature of the monolayer source is the discontinuity in the slope of the potential profile, changing abruptly (from 0.5 to $-0.5 \mathrm{~V} / \mathrm{m}$ for the uniform case) when crossing the monolayer in a direction parallel to its surface normal. Since $J_{z}=-\sigma \frac{\partial \Phi}{\partial z}$ and a unit value was taken for $J_{\Delta}$ as well as for $\sigma$, the observed slope reflects the symmetric outflow of one half of the surface current density from each side of the monolayer. Figure 4 is the quantification for the triangle of the general profiles of this fundamental source type as discussed in the major textbooks on potential theory (e.g., Figs. 1.7 in Panofski and Phillips [20]).

The dotted lines represent the potential profile generated by a single, equivalent monopole current source with strength $J_{\Delta} S_{\Delta}$. It illustrates that at sufficiently large distances from the source triangle, the potential may be approximated by that of a current monopole. The decay of the potential is as $1 / r$. However, close to the triangular source element the correspondence with the profile generated by the surface density is poor. The potential tends toward infinite values close to the point source. Depending on the accuracy aimed for, fields further away than, say, three times the size of the triangle expressed, e.g., by the radius of the circumscribed circle (2.07 for the triangle shown in Fig. 2) may permit field computations based on such equivalent sources.
Table 1 Columns $\Phi(\mathrm{A}), \Phi(\mathrm{B})$, and $\Phi(\mathrm{C})$ : the potential at vertices A, $\mathrm{B}$, and $\mathrm{C}$ of the source triangle specified in Fig. 2

\begin{tabular}{lllll}
\hline$J$ & $\Phi(\mathrm{A})$ & $\Phi(\mathrm{B})$ & $\Phi(\mathrm{C})$ & $\operatorname{Max}$ \\
\hline$\left[\begin{array}{lll}1 & 0 & 0\end{array}\right]$ & 0.06996 & 0.03794 & 0.05942 & 0.18814 \\
{$\left[\begin{array}{lll}0 & 1 & 0\end{array}\right]$} & 0.03323 & 0.08582 & 0.06954 & 0.19607 \\
{$\left[\begin{array}{lll}0 & 0 & 1\end{array}\right]$} & 0.03676 & 0.04788 & 0.12896 & 0.20679 \\
{$\left[\begin{array}{lll}1 & 1 & 1\end{array}\right]$} & 0.13992 & 0.17164 & 0.25791 & 0.45853 \\
\hline
\end{tabular}

The first three rows: linearly distributed monolayer, with the current source density $J$ at the vertices as specified in the first column, e.g., $\left[\begin{array}{lll}1 & 0 & 0\end{array}\right]$ : a local unit density at $\mathrm{A}$ and zero density at $\mathrm{B}$ and $\mathrm{C}$. The lower row lists the values for the uniform density, seen to be equal to the respective column sums, as required. The final column lists the extremes observed in the respective fields. These appear at different locations and, hence, the sum of its first three rows needs not to be equal to the value in the 4th row. Note that the values of diagonal terms of the inner $3 \times 3$ matrix are one half of the values in the lower row (the uniform case)

For the computation of the most significant terms in the BEM transfer matrix, the values at the vertices of the source triangles, such approximations are inadequate, as can be seen in Fig. 4.

In Appendix 3, dedicated analytical expressions are shown for the values at the vertices. These have been derived directly from Eqs. 19 and 20.

\subsection{Comparison with numerical integration}

Various algorithms have been published for solving integrals of scalar functions $f(\vec{r})$ over a triangle. These involve weighted sums of function evaluations at selected nodes on the triangle. Basic schemes are such as listed in Sect. 25.4 of [1], advanced ones such as in [26]. By increasing the number of nodes the accuracy of the results can be improved. However, if a closed form analytical solution is lacking the accuracy can not be quantified. This problem is more acute in cases where $f(\vec{r})$ is singular, such as in Eq. 6 . 
Fig. 3 As in Fig. 2, now with the plane of observation at $z=0.5$. Dotted the projections of the contours of the source triangle at $z=0$ (Fig. 2). Maxima left and right panels: 0.098542 and 0.27704 , respectively; step sizes left and right: 0.0125 and 0.025 , respectively

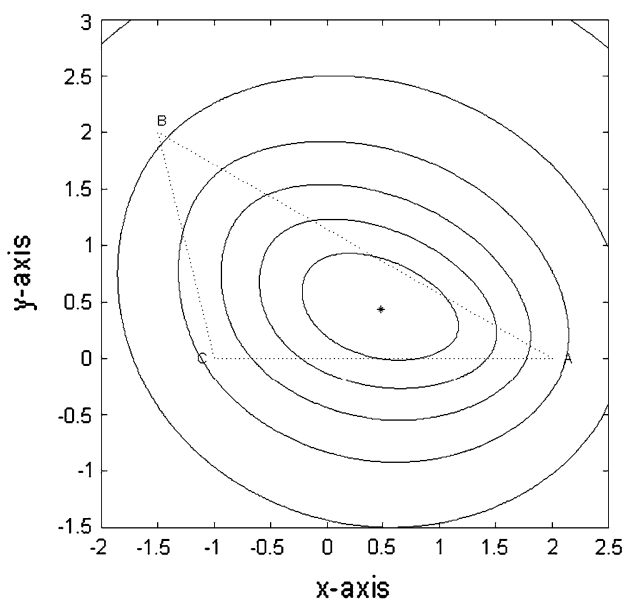

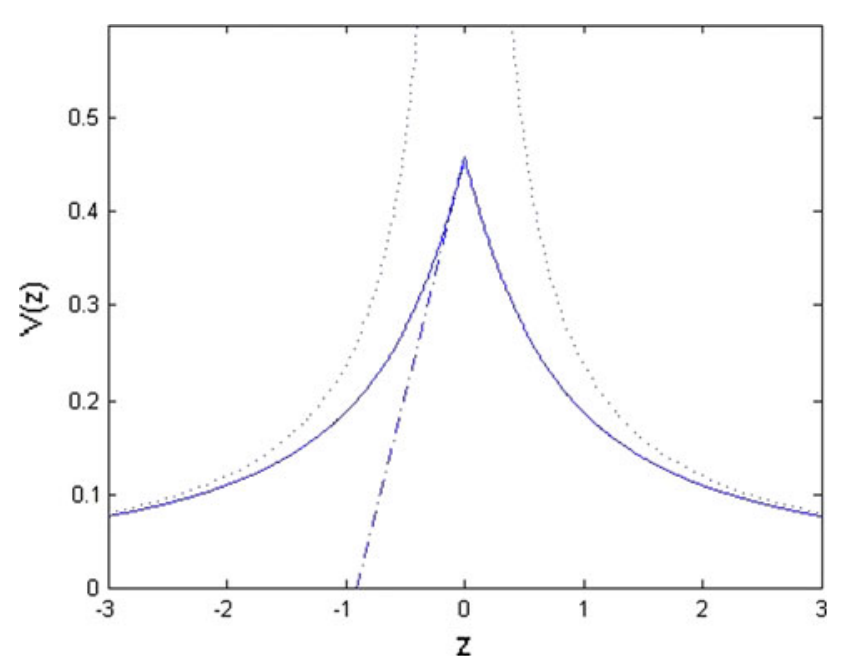

Fig. 4 Solid line potential profile along the axis parallel to the $z=$ axis, passing through the center of gravity (COG) of the source triangle (right panel Fig. 2). Uniform monolayer strength $J_{\Delta}=1$ $\mathrm{A} / \mathrm{m}^{2}$. Dotted line potential profile of a current monopole with strength $J_{\Delta} S_{\Delta}$ located at the COG. Dashed-dotted line the tangent to profile for $z \rightarrow 0$ (slope: $0.5 \mathrm{~V} / \mathrm{m}$ )

In such cases iterative refinements of the spatial sampling are used until a predefined convergence criterion is met. In BEM applications, the number of function calls required may be high and the "true" solution may not be found.

The performance of the Matlab function quad $2 d$, based on [26], using its default tolerance settings, was tested in its computation of Eq. 6, while taking the results based on Eq. 16, implemented by the author in a Matlab script $p d m l t r$, taken as the reference. Field points were used with their 3D coordinates drawn from the standard Gaussian density, which were shifted to the COG of the triangle shown in Fig. 2. In runs with 1000 of such random field points the maximum of the observed relative absolute errors was typically $0.05 \%$. The total computation time required for the 1000 calls of quad $2 d$ was $6.3 \mathrm{~s}$, when using pdmltr it was $0.23 \mathrm{~s}$, a time-ratio of about 28 . In runs where the field points were projected on the plane of the triangle, the maximum of the relative absolute errors was high as $0.5 \%$, the time-ratio increased up to 90 . When restricting the observation points to the vertices of the triangle, the maximum relative error was $5.5 \mathrm{e}-6$; the time-ratio was 3.3.

\section{Discussion}

In this article, a closed form analytical expression is presented for computing the potential field generated by a current monolayer current whose strength is linearly distributed on a triangle. The pertinent expressions are Eqs. 15 and 16. These imply the correct treatment of the singularity of the function $1 / r$ for coinciding source and field point locations. Expressions for handling this situation are shown in Appendix 3.

The derivation of Eq. 16 was inspired by a dedicated analysis [28] of de Munck's procedure for finding the corresponding solution for the linearly distributed double layer [6]. The implementation of Eq. 16 is less forbidding than may appear at first glance. The intermediate values of computations such as triangle edge lengths, normals, solid angles, and the logarithms are identical to the ones required for computing the field generated by a double layer on a triangle with linearly distributed strength (compare Eqs. 4, 15,25 , and 26).

Suggestions for using higher order shape functions have been reported in the literature, e.g., [14]. When considering their application on any given triangular mesh, one would be well advised to contrast this to a straightforward refinement of the triangulation in which all additional nodes are projections of the triangle refinement onto the actual, generally non-planar, geometry treated [31].

The interest in the use of the method of fundamental solutions (MFS) appears to be increasing [8]. As in early 
BEM applications [10], this method implies the use of sets of virtual monopolar sources, for which the infinite medium potential field is indeed fundamental and simple. However, by their nature these sources have an essential singularity at their locus (Fig. 4), which necessitates the inclusion of an extremely dense set of nodes in the handling of boundary value problems. This holds true in particular in situations where the boundaries of the nonintersecting interfaces of different compartments are close to one another. Statements made in the literature that the MFS would obviate the meshing of the involved surfaces conceal the fact that imaging the results demands the construction of such meshes.

The closed-form analytical expression presented in this paper is exact, may serve as the gold standard and is faster than numerical procedures. For large-scale applications [2] an implementation, e.g., in $\mathrm{C}^{++}$, will obviously yield timing values that differ from the ones listed.

The numerical values listed in Table 1 may serve to check any implementation of the basic expressions described. A basic Matlab script for the involved computations is available upon request from the author.

Open Access This article is distributed under the terms of the Creative Commons Attribution Noncommercial License which permits any noncommercial use, distribution, and reproduction in any medium, provided the original author(s) and source are credited.

\section{Appendix 1: The field produced by a line source}

The expressions for the field produced by source distributions over a triangle comprise terms that can be interpreted as the fields resulting from a line source. This problem is discussed here in some detail to provide a correct physical interpretation of such terms.

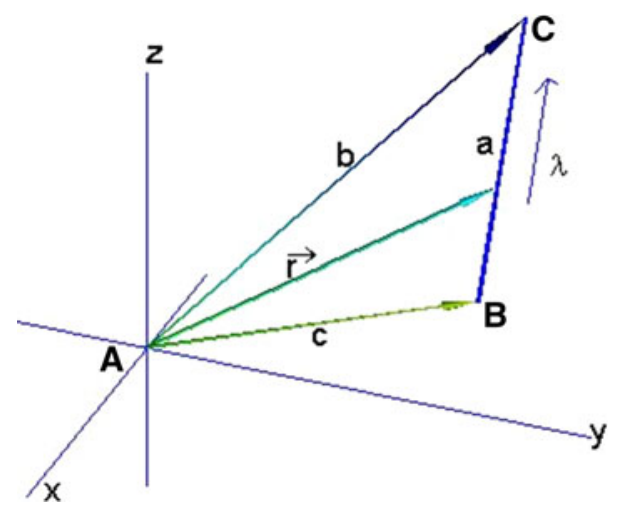

Fig. 5 Diagram introducing the computation of the potential at the origin generated by a line source density along a line segment with endpoints B and C. The vector $\vec{r}$ is drawn from the field point at the origin to an elementary part of the line source
The problem to be solved is illustrated in Fig. 5. The labels of the edge lengths of triangle $\mathrm{ABC}$ reflect those of the opposite vertices. A current line density $\tau$ (unit $\mathrm{A} / \mathrm{m}$ ) is impressed in a conducting infinite medium surrounding a line segment "a" with length $a$ having end points B and C relative to the field point $A$ at the origin. The potential at the origin, $\Phi(\mathrm{A})$, is found by integration of the contributions of point current sources at positions $\vec{r}$ along the line source segment, with strength $I(\vec{r})=\tau(\vec{r}) a \mathrm{~d} \lambda$ (unit A), in which $\lambda$ is a dimensionless integration variable. Accordingly, following (20.13), we have

$\Phi(\mathrm{A})=\frac{1}{4 \pi \sigma} \int_{0}^{1} \frac{\tau(\vec{r}) a}{r(\lambda)} \mathrm{d} \lambda$.

In the sequel, for ease of notation, the factor preceding the integral is dropped.

The uniform case

In the sequel, a uniform line source density is assumed with unit strength, i.e., $\tau(\vec{r})=1 \mathrm{~A} / \mathrm{m}$.

This leaves the following integral to be determined:

$I_{\mathrm{u}}=\int_{0}^{1} \frac{a}{r(\lambda)} \mathrm{d} \lambda$.

Note that this expression is invariant to an overall scaling of the geometry (Fig 5). The distance function $r(\lambda)$ can be expressed as $r(\lambda)=\sqrt{a^{2} \lambda^{2}+Q^{2} \lambda+c^{2}}$, with $Q^{2}=b^{2}$ $-a^{2}-c^{2}$.

The integral is a standard one. Using Dwight:380.001 listed in [7], we have

$$
\begin{aligned}
I_{\mathrm{u}} & =\left.\ln \left(2 a^{2} \lambda+Q^{2}+a r(\lambda)\right)\right|_{\lambda=0} ^{\lambda=1} \\
& =\ln \frac{(b+a)^{2}-c^{2}}{b^{2}-(c-a)^{2}} .
\end{aligned}
$$

On the basis of the triangle inequalities, both numerator and denominator in the fractions appearing in Eq. 19 are non-negative. Moreover, with the zero reference potential at infinity, the integral must be positive. Hence, the fractions forming the arguments of the logarithms are greater than one. Equation 19 holds true throughout 3D space; at field points coinciding with the line source its value is infinite.

The non-uniform case

For a line source density that varies linearly from $\mathrm{B}$ to $\mathrm{C}$ along edge $a$ we need to find $I_{\text {nu }}=\int_{0}^{1} \frac{c \lambda}{r(\lambda)} \mathrm{d} \lambda$.The solution 
to this integral is as listed in Dwight:380.011 [7] and can be expressed as

$I_{\mathrm{nu}}=\frac{b-c}{a}+\frac{a^{2}+c^{2}-b^{2}}{2 a^{2}} \ln \frac{(b+a)^{2}-c^{2}}{b^{2}-(c-a)^{2}}$.

The solution for the source strength increasing linearly from $\mathrm{C}$ to $\mathrm{A}$ is simply found by exchanging $c$ and $b$ in Eq. 20. This needs to be applied only to the constant term and the factor scaling the logarithm. The logarithm itself is unaffected. The summation of the two solutions yields the solution for the uniform situation (Eq. 19), as required.

\section{Appendix 2: The integral of $r(\lambda)$ over a line segment}

In the field problem discussed in Sect. 4, the integral over a line segment of $r(\lambda)$ appears, rather than that of its reciprocal value appearing in (18). Using the result listed as Dwight (3880.201) [7], and employing the same notation, the result is

$I_{1}=\left(2 b c^{2}+(b-a) Q^{2}-\frac{D}{2 c} I_{\mathrm{u}}\right) /(4 c)$,

with $D=Q^{4}-4 a^{2} c^{2}$ the discriminant of the parabolic expression in $r(\lambda)$.

\section{Appendix 3: The potentials at the vertices of the source triangle}

Equation 15 reduces for field points at the vertices of the source triangle. For a triangle with vertices, A, B, and C, with unit density at $\mathrm{A}$, and zero densities at $\mathrm{B}$ and $\mathrm{C}$ the reduced variants are listed in Eqs. 22a, 22b, 22c, and 22d. The edge lengths are labeled $a, b$, and $c$, corresponding to the opposite vertices $\mathrm{A}, \mathrm{B}$, and $\mathrm{C}$, as is commonly used in planar geometry.

$\Gamma(\mathrm{A})=\frac{h_{\mathrm{A}}}{2} \gamma$,

$\Gamma(\mathrm{B})=\frac{h_{\mathrm{A}}}{2}\left(\frac{c-b}{a}+\frac{b^{2}+a^{2}-c^{2}}{2 a^{2}} \gamma\right)$,

$\Gamma(\mathrm{C})=\Gamma(\mathrm{A})-\Gamma(\mathrm{B})$,

$\Gamma_{\text {uniform }}=h_{\mathrm{A}} \gamma=2 \Gamma(\mathrm{A})$,

with $h_{A}=\frac{2 S_{\Delta}}{a}$ the distance between vertex A and edge $a$, and $\gamma=\log \frac{(a+b)^{2}-c^{2}}{b^{2}-(c-a)^{2}}$. After scaling Eqs. 22a, 22b, 22c, and 22d, applied to the triangle specified in Fig. 2, by $\frac{1}{4 \pi}$, the results are those listed in column $\Phi(\mathrm{A})$ of Table 1 . The other two columns are found by cyclic permutation of the vertex and edge labels.
Appendix 4: The corresponding results for the double layer

As discussed in Sect. 1, some BEM applications involve source distributions of the double layer type. For the planar triangle, the potential field can be viewed as if generated by elementary current dipoles oriented normal to the source triangle (dipole strength: $d(\vec{r}) \vec{n}_{\mathrm{n}} \mathrm{d} S$.

The potential at the field point (shifted to the origin) follows from taking the integral of the contributions of elementary current dipoles directed along the surface normal, with dipole strength $d(\vec{r}) \vec{n}_{\mathrm{n}} \mathrm{d} S$ (unit Am). The result, with the source-field configuration shifted such that the field point lies at the origin, is [21],

$\Phi=\frac{1}{4 \pi \sigma} \int_{\Delta} \frac{\vec{r}}{r^{3}} \cdot \vec{n}_{\mathrm{n}} d(\vec{r}) \mathrm{d} S$.

with $d(\vec{r})$ the current dipole surface density (unit $\mathrm{Am} / \mathrm{m}^{2}=$ $\mathrm{A} / \mathrm{m})$. If this density is uniform, i.e., $d(\vec{r})=d$, the solution simplifies to

$\Phi=\frac{d}{4 \pi \sigma} \Omega_{\Delta}$,

with $\Omega_{\Delta}$ denoting the solid angle subtended by the triangle at the origin (as in Eq. 4) [21].

A numerically efficient and accurate expression for $\Omega_{\Delta}$, reads [30],

$\Omega_{\Delta}=2 \operatorname{atan} 2\left(\left[\overrightarrow{\mathrm{r}}_{\mathrm{k}} \overrightarrow{\mathrm{r}}_{1} \overrightarrow{\mathrm{r}}_{\mathrm{m}}\right], \mathrm{r}_{\mathrm{k}} \mathrm{r}_{1} \mathrm{r}_{\mathrm{m}}+\mathrm{r}_{\mathrm{k}} \overrightarrow{\mathrm{r}}_{1} \cdot \overrightarrow{\mathrm{r}}_{\mathrm{m}}+\mathrm{r}_{1} \overrightarrow{\mathrm{r}}_{\mathrm{m}} \cdot \overrightarrow{\mathrm{r}}_{\mathrm{k}}+\mathrm{r}_{\mathrm{m}} \overrightarrow{\mathrm{r}}_{\mathrm{k}} \cdot \overrightarrow{\mathrm{r}}_{1}\right)$,

in which ATAN2(Y,X) is Matlab's four quadrant arctangent of $\mathrm{X}$ and $\mathrm{Y}:-\pi<=\operatorname{ATAN} 2(\mathrm{Y}, \mathrm{X})<=\pi$ that takes into account the separate signs of the two arguments. This expression has the desirable property that its sign changes if the field point crosses the surface element [22].

Note that, as for the line source, Eq. 17, the potential is invariant to an overall scaling of the geometry, viz., the tetrahedron formed by the vertex indices of $\Delta$ and the field point (Fig. 1). In contrast, the corresponding expressions for the monolayer depend linearly on such scaling.

The solution for the linearly distributed double layer strength as derived by de Munck [6] shown below, using the notation used in this article is

$\omega=\frac{1}{n^{2}}\left(\mathrm{Z}^{\prime} \mathbf{n} \Omega_{\Delta}-\mathbf{E}_{\mathrm{c}}^{\prime} \mathbf{E}_{\mathrm{n}} \gamma T\right)$.

The three elements of the column vector $\omega$ refer to three linearly "weighted" solid angles, similar to those of the $\boldsymbol{\Gamma}$ weights described in Eq. 4 for the distributed monolayer. 


\section{References}

1. Abramowitz M, Stegun IE (1970) Handbook of mathematical functions. Dover, New York

2. Ataseven Y, Akahn-Acar Z, Akar CE, Gencer NG (2008) Parallel implementation of the accelerated BEM approach for EMSI of the human brain. Med Biol Eng Comput 46:671-679

3. Barr RC, Ramsey M, Spach MS (1977) Relating epicardial to body surface potentials by means of transfer coefficients based on geometry measurements. IEEE Trans Biomed Eng BME-24:1-11

4. Brebbia CA (1984) The boundary element method for engineers. Pentech Press, London

5. Colli-Franzone P, Guerri L, Taccardi B, Viganotti C (1980) Inverse epicardial mapping in the human case. In: Presented at proceedings symposium on the electrophysiology of the heart, New York

6. de Munck JC (1992) A linear discretization of the volume conductor boundary integral equation using analytically integrated elements. IEEE Trans Biomed Eng BME-39:986-990

7. Dwight BH (1961) Tables of integrals and other mathematical data. MacMillan, New York

8. Fairweather G, Karageorghis A (1998) The method of fundamental solutions for elliptic boundary value problems. Adv Comput Math 9:69-95

9. Ferguson AS, Zhang X, Stroink G (1994) A complete linear discretization for calculating the magnetic field using the boundary element method. IEEE Trans Biomed Eng BME-41: 455-460

10. Gelernter HL, Swihart JC (1964) A mathematical-physical model of the genesis of the electrocardiogram. Biophys J 4:285-301

11. Graglia RD (1993) On the numerical integration of the linear shape functions times the 3-D green's function or its gradient on the plane triangle. IEEE Trans Antennas Propag 41:144-1455

12. Gulrajani RM (1998) Bioelectricity and Biomagnetism. Wiley, New York

13. Gulrajani RM (ed) (1998) The forward problem in electrocardiography. In: Bioelectricity and biomagnetism. Wiley, New York, pp. $348-380$

14. Kuwahara T Tadeka T (1987) A formula of boundary integral for potential problem and its consideration. In: Presented at proceedings of the 1st Japan-China symposium on boundary element methods

15. Kuwahara T, Takeda T (1986) An effective analysis for threedimensional boundary element method using analytical higher order elements. Trans IEE Jpn 107-A:275-282

16. Lynn MS, Timlake WP (1968) The numerical solution of singular equations of potential theory. Numer Math 11:77-98
17. Martin RO (1970) Inverse electrocardiography, $\mathrm{PhD}$, Duke University, Duke, NC, USA

18. Medina DE, Liggett JA (1989) Exact integrals for the threedimensional boundary element potential problems. Commun Appl Numer Anal 5:555-561

19. Okon EE, Harrington RF (1982) The potential due to a uniform source distribution over a triangular domain. Int J Numer Method Eng 18:1401-1411

20. Panofski WKH, Phillips M (1962) Classical electricity and magnetism. Addison-Wesley, London

21. Plonsey R (1969) Bioelectric phenomena. McGraw-Hill, New York

22. Plonsey R, van Oosterom A (2011) Introductory physics and mathematics. In: Macfarlane PW, van Oosterom A, Pahlm O, Kligfield P, Janse MC, Camm J (eds) Comprehensive Electrocardiology, vol 1, 2nd edn. Springer, London, pp 51-101

23. Potse M, Dube B, Vinet A (2009) Cardiac anisotropy in boundary-element models for the electrocardiogram. Med Biol Eng Comput 47:719-729

24. Rao SM, Glisson AW, Wilton DR, Vidula BS (1979) A simple numerical solution procedure for statics problems involving arbitrary-shaped surfaces. IEEE Trans Antennas Propag AP-36: 604-608

25. Rudy Y, Burns JE (1999) Noninvasive electrocardiographic imaging. Ann Noninvasive Electrocardiol 4:340-359

26. Shampine LF (2008) Matlab Program for Quadrature in 2D. Appl Math Comp 202:266-274

27. Smythe WR (1968) Static and dynamic electricity. McGraw-Hill, New York

28. van Oosterom A (2012) Potential fields of boundary element methods. In: Barr RC (ed) The biomedical engineering handbook, vol 4, 4th edn. CRC Press, New York

29. van Oosterom A, Oostendorp TF (1992) On computing pericardial potentials and current densities. J Electrocardiol 25(Suppl): $102-106$

30. van Oosterom A, Strackee J (1983) The solid angle of a plane triangle. IEEE Trans Biomed Eng BME-30:125-126

31. Zhou H, van Oosterom A (1993) Mesh refinement and accuracy of numerical solutions. In: Presented at engineering solutions to current health care problems. Proceedings of the XVth annual international conference of the IEEE Engineering in Medicine and Biology Society, Piscataway

32. Zhou H, van Oosterom A (1994) Application of the boundary element method to the solution of anisotropic magnetic problems. Med Biol Eng Comput 32:399-405 